\title{
Battery Management System for Future Electric Vehicles
}

\author{
Bedatri Moulik $^{1, *}$ and Dirk Söffker ${ }^{2}$ (I) \\ 1 Department of Electrical and Electronics Engineering, Amity University, Sector 125, Noida 201313, India \\ 2 Chair of Dynamics and Control, University of Duisburg-Essen, Lotharstrasse 1-21, \\ 47057 Duisburg, Germany; soeffker@uni-due.de \\ * Correspondence: bmoulik@amity.edu
}

Received: 7 July 2020; Accepted: 21 July 2020; Published: 24 July 2020

\begin{abstract}
The future of electric vehicles relies nearly entirely on the design, monitoring, and control of the vehicle battery and its associated systems. Along with an initial optimal design of the cell/pack-level structure, the runtime performance of the battery needs to be continuously monitored and optimized for a safe and reliable operation and prolonged life. Improved charging techniques need to be developed to protect and preserve the battery. The scope of this Special Issue is to address all the above issues by promoting innovative design concepts, modeling and state estimation techniques, charging/discharging management, and hybridization with other storage components.
\end{abstract}

Keywords: battery electric vehicles; battery management; hybrid energy storage

\section{Introduction}

Recent advancements in battery technology have pushed the sales of electric (EVs) and hybrid electric vehicles (HEVs) further. The improvements in the EV/HEV range, energy/charging efficiency, safety, reliability, and lifetime are entirely dependent on the design and chemistry of the battery pack and its associated systems. Most of the safety concerns regarding the battery's unexpected temperature rise and predictions of the internal reactions leading to fluctuations in internal temperature also need to be addressed.

\section{Battery Management System for Future Electric Vehicles}

The aim of the Special Issue "Battery Management System for Future Electric Vehicles" is to investigate advanced battery management technologies for the estimation, monitoring, and control of battery states, associated modeling techniques, thermal and charging/discharging management for optimized life, performance, and range. Optimal sizing, the hybridization of storage systems, and innovative battery test-benches were also encouraged. There are a total of seven accepted and published papers, which are summarized as follows:

The first paper, authored by Hakeem and Solyali [1], presents a battery thermal management system (BTMS) with improved performance in terms of battery cooling. An improved pack structure is proposed, which is experimentally investigated with different air flow rates and current rates of charge-discharge profiles. Finally, based on the obtained data, an artificial neural network is trained to obtain the thermal model of the battery pack.

The second paper, authored by Tseng and Yang [2], presents a torque and battery distribution strategy (TBD) that takes into account the torque-speed characteristics, as well as the battery state of charge to obtain optimized range and efficiency. Based on the State of Charge (SoC) gaps and ratios between the front and the rear battery packs, three torque distribution modes are then 
proposed. First simulation, then hardware-in-the-loop experimentation, followed by actual road tests, are performed to validate the effectiveness of the TBD in the extension of the electric vehicle range.

The third paper, authored by Kuo [3], presents a battery model based on a modified Thévenin circuit, Butler-Volmer kinetics, Arrhenius equation, Peukert's law, and a back propagation neural network (BPNN). The model can estimate the coulombic efficiency and the remaining capacity of the battery, as analyzed experimentally under various environmental conditions. Based on experimental results and curve fitting techniques, a comprehensive model is developed. A correction factor is introduced and the prediction of remaining capacity is done using a BPNN.

The fourth paper, authored by Cao [4], presents a wireless distributed and enabled battery energy storage system (WEDES) for electric vehicles (EVs) derived using a small signal modeling technique. The WEDES controller is designed to address SoC balancing, bus voltage regulation, and battery module current/voltage regulation at the same time. Finally, simulation and hardware experiments are carried out to evaluate and validate the accuracy and effectiveness of the derived model and controller.

The fifth paper, authored by Guo, et al. [5], presents an online SoC estimation method by using an equivalent circuit model, followed by model parameter identification. An optimization method is proposed to improve the accuracy of the SoC estimation. Then, an online estimation based on the adaptive unscented Kalman filter (AUKF), and with optimized model parameters, is performed. The estimation accuracy of the AUKF with the UKF is compared. The convergence of the initial error of the AUKF before and after parameter optimization is also compared.

The sixth paper, authored by Chen, Chen, and Duan [6], presents an optimization method to cooperatively optimize the economic dispatching and capacity allocation of both renewable energy sources (RESs) and electric vehicles (EVs). Both the installation capacity of RESs and the number of EV charging/discharging infrastructures (EVCDIs) are optimized. This optimization method is based on the EVs' across-time-and-space energy transmission. The main optimization objective is to improve the economics of the system allocation and decrease the cost of the microgrid operator. A two-loop optimization is considered using an improved particle swarm optimization (IPSO). The inner loop comprises the optimization of system dispatching, while in the outer loop, the allocation of EVs and RESs is optimized.

The seventh paper, authored by Hou, et al. [7], presents a variational Bayesian approximation-based adaptive dual extended Kalman filter (VB-ADEKF) to improve the accuracy of SoC estimation. First, the variational Bayesian results are used along with the extended Kalman filter to jointly estimate the states. Next, both variational Bayesian and variational Bayesian approximation-based adaptive dual extended Kalman filters are alternatively used. Additionally, measurement noise variances are considered to compensate for uncertainties in measurement. With the help of experiments, the proposed VB-ADEKF algorithm is compared with the traditional DEKF algorithm in terms of SoC estimation accuracy, convergence rate, and robustness.

Thus, summarizing all the seven papers brings us to the conclusion that this Special Issue has been successful in bringing together novel contributions considering multiple aspects of energy storage management and optimized charging/discharging schedules.

\section{Future Battery Management Systems}

Although this Special Issue is finished, immense work still remains in the field of innovative battery state estimation algorithms and optimization approaches to improve their accuracy and reliability in terms of online and real-time application in EVs.

Author Contributions: Both authors contributed equal parts to the paper, whereby the corresponding author was mainly responsible for initial writing, the second author for structuring and organizing, reviewing, and summarizing the entire contribution. All authors have read and agreed to the published version of the manuscript.

Funding: This research received no external funding. 
Acknowledgments: This Special Issue would not have been a success without the constant efforts of the authors-including those whose papers could not be selected. We can only hope that this Special Issue is able to provide them with a platform to learn and improve their existing works based on the valuable comments of the reviewers and editors. We would also like to thank the reviewers for dedicating their time and energy to the submitted manuscripts and motivating the authors to improve their work. Last, but not least, we would like to extend our heartiest congratulations to the entire editorial team of Applied Sciences for their sincere efforts and constant dedication in making this Special Issue a success.

Conflicts of Interest: The authors declare no conflict of interest.

\section{References}

1. Hakeem, A.A.; Solyali, D. Empirical Thermal Performance Investigation of a Compact Lithium Ion Battery Module under Forced Convection Cooling. Appl. Sci. 2020, 10, 3732. [CrossRef]

2. Tseng, Y.H.; Yang, Y.P. Torque and Battery Distribution Strategy for Saving Energy of an Electric Vehicle with Three Traction Motors. Appl. Sci. 2020, 10, 2653. [CrossRef]

3. Kuo, T.J. Development of a Comprehensive Model for the Coulombic Efficiency and Capacity Fade of LiFePO4 Batteries under Different Aging Conditions. Appl. Sci. 2019, 9, 4572. [CrossRef]

4. Cao, Y. Small-Signal Modeling and Analysis for a Wirelessly Distributed and Enabled Battery Energy Storage System of Electric Vehicles. Appl. Sci. 2019, 9, 4249. [CrossRef]

5. Guo, X.; Xu, X.; Geng, J.; Hua, X.; Gao, Y.; Liu, Z. SOC Estimation with an Adaptive Unscented Kalman Filter Based on Model Parameter Optimization. Appl. Sci. 2019, 9, 4177. [CrossRef]

6. Chen, J.; Chen, C.; Duan, S. Cooperative optimization of electric vehicles and renewable energy resources in a regional multi-microgrid system. Appl. Sci. 2019, 9, 2267. [CrossRef]

7. Hou, J.; Yang, Y.; He, H.; Gao, T. Adaptive dual extended Kalman filter based on variational bayesian approximation for joint estimation of lithium-ion battery state of charge and model parameters. Appl. Sci. 2019, 9, 1726. [CrossRef]

(C) 2020 by the authors. Licensee MDPI, Basel, Switzerland. This article is an open access article distributed under the terms and conditions of the Creative Commons Attribution (CC BY) license (http://creativecommons.org/licenses/by/4.0/). 